\title{
A study of the frequency characteristics of a photovoltaic convertor PPC-4E
}

\author{
Jovan Shikoski, ${ }^{* 1}$ Rumen Arnaudov, ${ }^{1}$ and Tinko Eftimov ${ }^{2}$ \\ ${ }^{I}$ Technical University of Sofia, $8 \mathrm{Kl}$. Ohridski Blvd, Sofia 1700, Bulgaria \\ ${ }^{2}$ Université du Québec en Outaouais, Canada
}

Received September 03, 2018; accepted September 06, 2018; published September 30, 2018

\begin{abstract}
The paper considers the possibility to use a fibercompatible photovoltaic converter PPC-4E simultaneously as a detection photodetector for combined transmission of photonic power and optical communication signals along a single fiber. We have studied the frequency transfer characteristics of specialized converter PPC-4E designed primarily for photovoltaic conversion.
\end{abstract}

It is well known that optical fibers can transfer not only communication data but photonic energy which is converted to electrical at fiber output by means of a fibercoupled photovoltaic converter [1]. The advantages of photonic energy transmission are the immunity to electromagnetic interference, short-circuiting, sparking [2]. In addition, optical fibers are smaller in size and due to lower losses per unit length can transfer energy over considerable distances [3]. Optical cables are more resistant to humidity and corrosion [4]. The technology is used for systems of remote control, for powering of sensing networks and even for medical applications [5, 6].

In the present paper we present the results concerning the demodulation detecting characteristics of a fibercompatible photovoltaic power converter PPC-4E, which data are unavailable from the manufacturers [7]. The final objective is to simultaneously transmit both photonic power and communication data over a single fiber. The studies performed showed that the photovoltaic converter can detect signals of up to $3 \mathrm{MHz}$ modulation frequency. This leads to the conclusion that apart from being used as a photovoltaic converter the PPC-4E can simultaneously be used as an optical communication data detector.

The set-up for simultaneous transmission of photonic energy and communication data along a single fiber to the photovoltaic converter is shown in Fig. 1.

The laser driver is powered by a $12 \mathrm{~V} / 1.2 \mathrm{~A}$ power supply to allow for $500 \mathrm{~mW}$ optical power.

\section{E-mail: jovanshiko@abv.bg \\ E-mail: ra@tu-sofia.bg}

E-mail: tinko.eftimov@uqo.ca

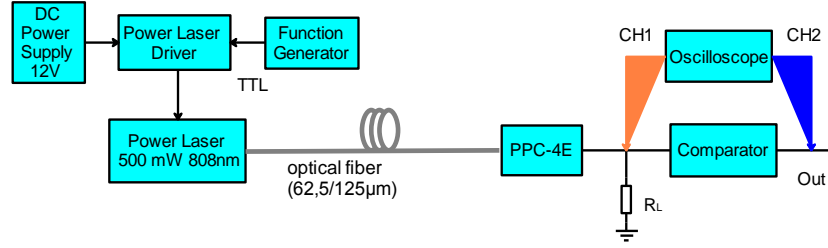

Fig.1. Optical set-up for the study of the frequency characteristics of a PPC-4E photovoltaic converter.

The functional generator provides a series of TTL signals at a given repetition frequency, which are applied to the digital input of a laser driver to modulate a high power laser diode emitting at $808 \mathrm{~nm}$. A $62.5 \mu \mathrm{m}$ multimode optical fiber of $55 \mathrm{~m}$ length transmits a $500 \mathrm{~mW}$ optical signal to the PPC-4E photovoltaic converter. The load resistance $R_{L}=50 \Omega$ is connected to its output. The voltage across the resistance is the output signal from the photovoltaic convertor and is next applied to channel 1 (CH1) of an oscilloscope. An increase in the frequency causes an increase in signal distortion, which leads to an increase in the bit error rate (BER). A comparator is then used to regenerate the form of the initial signal that is connected to channel $2(\mathrm{CH} 2)$. The comparison between the signal from the photovoltaic output $(\mathrm{CH} 1)$ and the regenerated signal $(\mathrm{CH} 2)$ permits to estimate its quality.

Distortions of the front shape and the phase of the signal at the photodetector are caused by processes of generation, diffusion and those of the carriers in the semiconductor. The distortions of the output signal from the detector increase with the modulation frequency. In Fig. 2 is shown the output signal from the converter (CH1) and that from the output of the comparator $(\mathrm{CH} 2)$. For standard frequencies up to $400 \mathrm{kHz}$ no significant distortions are observed and no bit errors arise. At $460.8 \mathrm{kHz}$ (Fig. 2) weak distortions in the upper front part of the output pulse from the PPC are observed, while those from the comparator remain unchanged. At $1 \mathrm{MHz}$ (Fig. 3) the rise time increases, which decreases the slope of the rise front of the pulse from the PPC. 


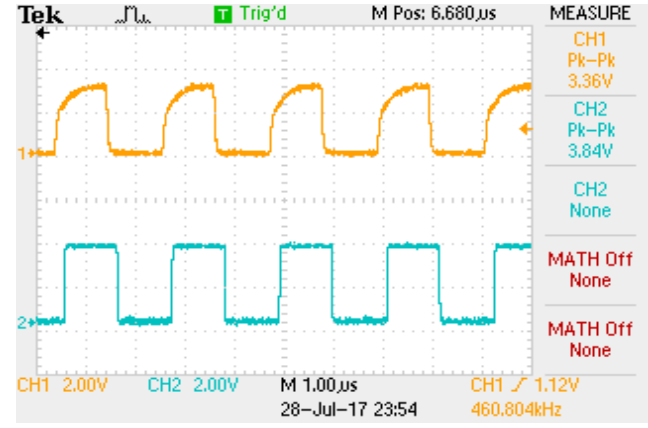

Fig. 2. Output signals from the PPC-4E and the comparator at $460.8 \mathrm{kHz}$.

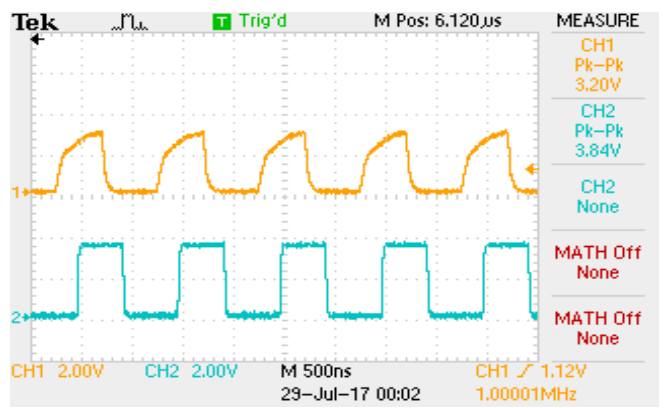

Fig. 3. Output signals from the PPC-and the comparator at $1 \mathrm{MHz}$.

The signal shape from the comparator remains unchanged, but a phase shift of $45^{\circ}$ with respect to the PPC is observed.

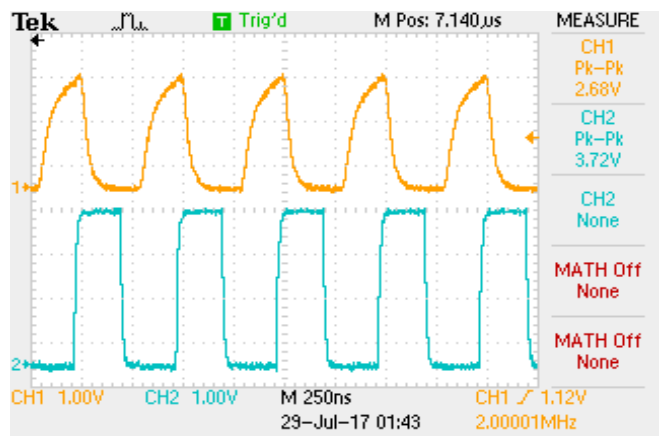

Fig. 4. Output signal from PPC-4E and the comparator at $2 \mathrm{MHz}$

At a frequency of $2 \mathrm{MHz}$ (Fig. 4) the PPC generates practically triangular pulses but the signal from the comparator maintains a rectangular shape, the phase shift being $90^{\circ}$. At a frequency of $3 \mathrm{MHz}$ (Fig. 5) the distortion of the output signal from the PPC is further increased. Although at this frequency weak distortions of the regenerated signal compared to those from the comparator are observed, the pulses ' 1 ' and ' 0 ' are well defined. The phase difference between both signals is $180^{\circ}$.

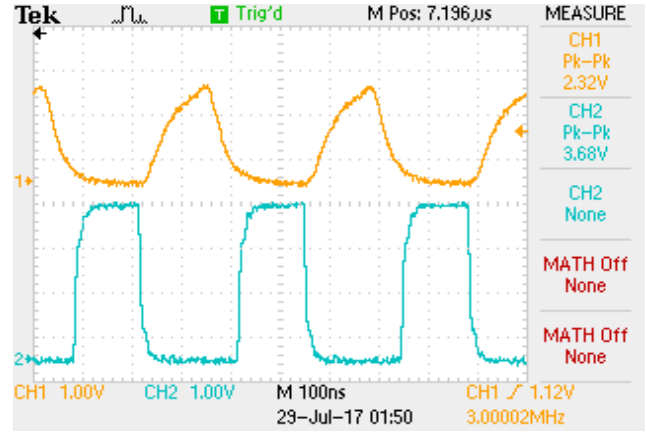

Fig. 5. The output signal from the PPC-4E and from the comparator at $3 \mathrm{MHz}$.

The results show that for frequencies up to $3 \mathrm{MHz}$ the signals generated by the PPC can successfully be regenerated by the comparator, and the PPC-4E can be used as a photodetector (photoreceiver).

The frequency response $f_{\mathrm{c}}$ at $-3 \mathrm{~dB}$ can be evaluated from the rise time $\tau_{\mathrm{r}}$ from 0.1 to 0.9 level, as $f_{\mathrm{c}}=0.35 / \tau_{\mathrm{r}}$ [9]. From Fig. 2 we can evaluate $\tau_{\mathrm{r}} \approx 0.35 \mu \mathrm{s}$, which yields $f_{\mathrm{c}}$ $\approx 1 \mathrm{MHz}$, at which we obtain a $45^{\circ}$ phase shift between the comparator output and thephotovoltaic output.

An important characteristic of a communication system is the probability for bit error (Bit Error Rate - BER) [8].

Fig. 6 shows the scheme of an experimental set-up for BER measurement of the PPC-4E convertor.

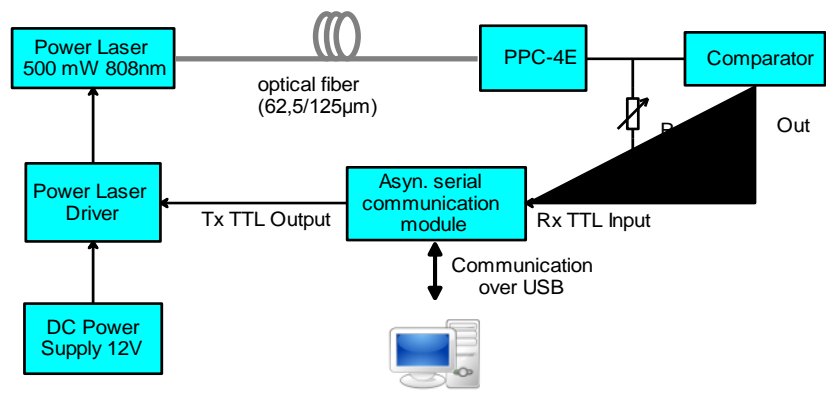

Fig. 6. Experimental set-up for BER measurement.

Using a UART module, the transmission of data is performed serially and asynchronously along an optical fiber. The UART module is linked via an USB port to a computer to measure the BER. Asynchronous transmission avoids the problems associated with the phase shifts of signals. The computer transmits through an asynchronous serial communication module a sequence of 10 bits: 0010110101 . The letter ' $Z$ ' is chosen because its ASCII represents a series ' 1 's and ' 0 's combined with a change in the signal phase shift of the last four bits with respect to the first four bits. A sequence of $32 \mathrm{MB}$ is transmitted to the input of the driver. The optical signals 
from the high power laser are transmitted to the PPC-4E module via a multimode fiber. The output signal is picked up from the load resistance $\mathrm{R}_{\mathrm{L}}$ of variable value whose shape is regenerated by the comparator. The regenerated signal from the comparator is applied to the receiver of the asynchronous communication module. The computer compares the transmitted to the received bits in every byte in a sequence of 3.2.MB. At the end of transmission of the whole series of bits, the computer, after comparing the transmitted and received symbols, counts the number of errors.

Table 1. The number of bit error for different loads.

\begin{tabular}{|l|c|c|c|c|}
\hline \multirow{2}{*}{$\begin{array}{c}\text { BER for a } \\
\text { load } R_{L}\end{array}$} & \multicolumn{5}{|c|}{ Bit Rate, Kbit/s } \\
\cline { 2 - 5 } & 230.4 & 460.8 & 576.0 & 960.0 \\
\hline $\mathrm{R}_{\mathrm{L}}=1000 \Omega$ & 0 & 0 & $2\left(5.96 \times 10^{-8}\right)$ & $20\left(59.6 \times 10^{-8}\right)$ \\
\hline $\mathrm{R}_{\mathrm{L}}=500 \Omega$ & 0 & 0 & $3\left(8.94 \times 10^{-8}\right)$ & $10\left(29.8 \times 10^{-8}\right)$ \\
\hline $\mathrm{R}_{\mathrm{L}}=100 \Omega$ & 0 & 0 & $2\left(5.96 \times 10^{-8}\right)$ & $5\left(14.9 \times 10^{-8}\right)$ \\
\hline $\mathrm{R}_{\mathrm{L}}=50 \Omega$ & 0 & 0 & 0 & $1\left(2.98 \times 10^{-8}\right)$ \\
\hline $\mathrm{R}_{\mathrm{L}}=25 \Omega$ & 0 & 0 & 0 & $3\left(8.94 \times 10^{-8}\right)$ \\
\hline $\mathrm{R}_{\mathrm{L}}=10 \Omega$ & 0 & 0 & $58\left(172.8 \times 10^{-8}\right)$ & - \\
\hline
\end{tabular}

The BER measurements were performed at frequencies standard for microcontrollers: $230.4 \mathrm{kHz}, 460.8 \mathrm{kHz}$, $576 \mathrm{kHz}$ and $960 \mathrm{kHz}$.

No measurements were performed for frequencies above $960 \mathrm{kHz}$ since the asynchronous serial communication module does not support higher frequencies. The number of bit errors is presented in Table 1. From the results presented it can be concluded that the maximum bit rate at which the photovoltaic converters can receive reliably data at $960 \mathrm{kbit} / \mathrm{s}$ for a load of $50 \Omega$

The BER is directly related to the SNR (Signal-to-NoiseRatio) since the reason for the appearance of bits are noises of various nature. The eye-diagram shows the noise level and the jitter. Figure 7 presents the eye-diagram of the photovoltaic using MATLAB visualisation for the reception of signals at $960 \mathrm{kbit} / \mathrm{s}$. We see that the eye is open at a maximum which implies that the noise level and the time jitter are low.

This shows that the PPC-4E can be used as a photodetector for the reception of data of rates up to about $1 \mathrm{Mbit} / \mathrm{s}$.

As a practical proof of the studies, a test was performed in which a multiple sequence of the following Albert Einstein's quotation was transmitted: "No amount of experimentation can ever prove me right; a single experiment can prove me wrong". The text was received and exhibited on the screen without any error at a bit rate of $960 \mathrm{kbit} / \mathrm{s}$.

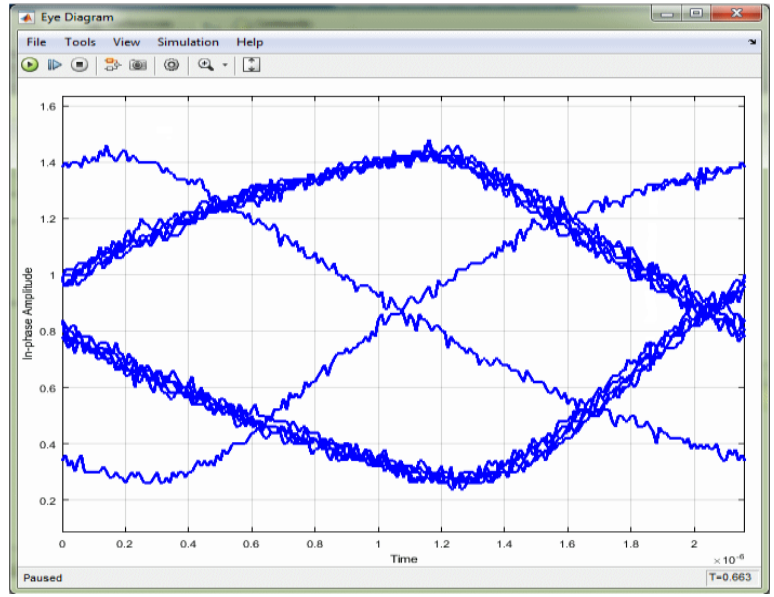

Fig. 7. Eye pattern for a bit rate of $960 \mathrm{kbit} / \mathrm{s}$.

In conclusions, the experiments performed allow us to conclude that the photovoltaic converter PPC-4E can detect signals moduated at frequencies of up to $3 \mathrm{MHz}$ for a load of $R_{L}=50 \Omega$. At this frequency the output signal can be regenerated by a comparator but phase shifted by $180^{\circ}$, which must be accounted for at the receiver. The measured BER for a variety of bit rates and load resistances shows that the photovoltaic converter PPC-4E can receive data for a load of $50 \Omega$ at rates of up to $960 \mathrm{kbit} / \mathrm{s}$, i.e. about $1 \mathrm{Mbit} / \mathrm{s}$. The measured BER is $2.98 \times 10^{-8}$.

The studies performed show that the fiber-compatible photovoltaic converter PPC-4E can simultaneously be used to convert photonic into electric energy and detect optical signals at a rate up to $1 \mathrm{Mbit} / \mathrm{s}$ along a single optical fiber.

\section{References}

[1] Powering Remote Data Links over Fiber, www.lumentum.com, Lumentum LLC, 2015.

[2] A. Basanskaya, IEEE Spectrum 40(10), 18 (2005).

[3] M. Cohen, OPTO and Laser Europe, 27-29 (2006).

[4] T. Yasui, J. Ohwaki, M. Mino, T. Sakai, 28th Photovoltaic Specialists Conference, 1614-1617 (2000).

[5] J.G. Werthen, Opt. Fiber Comm./Nat. Fiber Opt. Eng. Conf., 1-3 (2008).

[6] K. Iniewski, Medical Imaging: Principles, Detectors and Electronics, p. 292, New Jersey, 2009.

[7] Power-Over-Fiber Kit, www.lumentum.com, Lumentum LLC, 2015.

[8] S. Kartalopoulos, Optical Bit Error Rate: An Estimation Methodology (Willey- IEEE Press 2004).

[9] Electronic Devices and Circuits: Discrete and integrated in D.J. Daily Active filters (Prentice Hall 2001). 